\title{
Soft robotics: the route to true robotic organisms
}

\author{
Jonathan Rossiter ${ }^{1}$
}

Received: 11 January 2021 / Accepted: 9 June 2021 / Published online: 29 June 2021

(C) The Author(s) 2021

\begin{abstract}
Soft Robotics has come to the fore in the last decade as a new way of conceptualising, designing and fabricating robots. Soft materials empower robots with locomotion, manipulation, and adaptability capabilities beyond those possible with conventional rigid robots. Soft robots can also be made from biological, biocompatible and biodegradable materials. This offers the tantalising possibility of bridging the gap between robots and organisms. Here, we discuss the properties of soft materials and soft systems that make them so attractive for future robots. In doing so, we consider how future robots can behave like, and have abilities akin to, biological organisms. These include huge numbers, finite lifetime, homeostasis and minimaland even positive-environmental impact. This paves the way for future robots, not as machines, but as robotic organisms.
\end{abstract}

Keywords Soft robotics $\cdot$ Robot organisms $\cdot$ Biodegradable robots $\cdot$ Avogadro's number of robots

\section{Introduction}

Robots have been around for almost 100 years, from the earliest conceptualizations of L Frank Baum, the coining the of the term "robot" by Karel Čapek and the first industrial robots of Pollard and Roselund in the 1930's [1]. Robots are used widely in manufacturing [2], medicine [3], cooperative activities and space exploration [4]. Most robots have been designed and fabricated to a few very defined formulae. Typically, they are based on the human form (they are humanomimetic [5] robots), copy the functions of animals (they are zoomimetic [6]) or are defined from established mechanical principles derived from industrial revolution. While these approaches have helped build the global robotics sector into a more then $\$ 100$ bn concern, there is a hard limit as to what these robots can do.

Conventional robots are fabricated from largely rigid materials which, although aiding in control and determinism, constrain their range of motions and practical applications.

This work was presented in part as a plenary speech at the 26th International Symposium on Artificial Life and Robotics (Online, January 21-23, 2021).

Jonathan Rossiter

Jonathan.Rossiter@bristol.ac.uk

1 Department of Engineering Mathematics and Bristol Robotics Laboratory, University of Bristol, Bristol BS8 1TW, UK
For example, a rescue robot fabricated from rigid materials will inevitably get stuck if it enters a collapsed building. Conventional robots employ traditional electromagnetic actuation technologies which are costly and complex to make and limit scale-down. Consideration of toxicity and long-term environmental impact is secondary to the immediate task at hand-many robots employ materials that are toxic to the natural environment. The consequence of this toxicity is that any robot released into the environment must be captured and returned for safe recycling or disposal at end-of-life. This severely limits the number of robots that can be safely released into the environment. These limitations highlight how conventional robotics, despite an outward element of biomimetics, operate very far from the concepts that are so embedded in biological organisms. In contrast, Soft Robotics has sought to address these challenges and has increased significantly over the last decade, with major developments in autonomous robotics [7] smart skins [8] soft computation [9] and energy autonomy [10].

In this paper, we discuss the potential of soft robotics to deliver a step-change in robotics by bridging the gap between organic capabilities and engineered systems. The materials, mechanical structures and design flexibility of soft robotics can overcome many of the limitations of current robotic systems, including low numbers, low environmental compatibility, reliance on stored energy and low physical adaptability. In the next section, we will review the differences between hard and soft robots, pointing to barriers for 


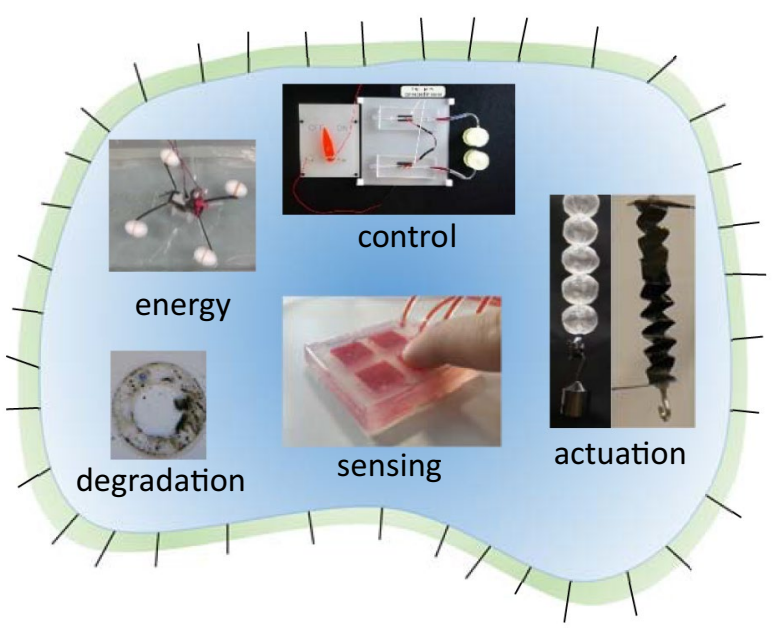

Fig. 1 A robotic organism has features of autonomous control [9], energy autonomy [21], sensing and actuation [26], and biodegradation at end-of-life [18]

development and opportunities for radical new thinking of what a robot really could be. We will then consider that future robots should not be thought of as machines, but as robotic organisms (Fig. 1). This re-focus of design and research effort provides a strong paradigm for rapid and continuous development of future robots and significantly expands their application. Finally, we will discuss current limitations and routes to realizing a future with ubiquitous soft robotic organisms.

\section{Rigid vs soft}

Table 1 contrasts the main capabilities of conventional hard robots and their soft counterparts. While there are exceptions to these general rules, this illustrates how scale-up of robotics must go together with important characteristics, such as biodegradability and low cost. These are achievable most readily with soft robotics. In contrast, ready controllability and high strength remain advantages of rigid robots.

\subsection{Large numbers}

Populations of robots have been used in many swarm studies, with over 1000 individual units operating together. These conventional robots-employing motors and silicon computation-are practically limited in numbers to $10^{5}-10^{6}$ due to their size, complexity, cost and rigidity. At smaller scales, nanoparticle swarms have been proposed and developed which can be injected into the body to locate and kill
Table 1 Conventional (hard) vs soft robotic characteristics

\begin{tabular}{lcc}
\hline & Hard & Soft \\
\hline Toxic & $\checkmark$ & $x$ \\
Large $\left(>10^{9}\right)$ numbers & $x$ & $\checkmark$ \\
Easy control & $\checkmark$ & $x$ \\
Biodegradable & $x$ & $\checkmark$ \\
Bio-integrative & $x$ & $\checkmark$ \\
Self-repairing & $x$ & $\checkmark$ \\
Low-cost & $x$ & $\checkmark$ \\
Environment energy use & $\checkmark$ & $\checkmark$ \\
High strength & $\checkmark$ & $x$ \\
Novel actuation & $x$ & $\checkmark$ \\
\hline
\end{tabular}

cancer [11]. These have the potential to be manufactured at numbers exceeding Avogadro's constant $\left(6.02 \times 10^{23}\right)$ but are functionally simple structures and cannot be termed robots. In contrast, soft robots have the potential to be fabricated in numbers far beyond those of conventional robotics. These small soft robots can be made at the scales of biological microorganisms and bacteria, with populations potentially exceeding $10^{10}$ within the next decade. This represents a significant opportunity, especially when viewed in the context of the estimated $3 \times 10^{6}$ total number of industrial robots operating in 2020 .

\subsection{Controllability}

Controllability is a concept that has very different meanings depending on the complexity of individual robots and the number of robots operating together. Soft robotics can be fabricated in very large numbers, with each individual having relatively simple capabilities. Swarm-based control approaches dominate, with the challenge becoming one of stochastic determinism and prediction of emergent behaviours [12]. This mitigates the challenges of controlling larger multi-degree of freedom (DOF) soft robotics [13] since the individual soft robots can have low DOF. However, each robot still requires the capability to locally compute and undertake reactive control tasks. To partially overcome this limitation, computation can be devolved to the environment: by exploiting chemical message passing-akin to ant pheromones-, memory, control and population-level behaviours can be realised [14].

Recently, non-silicone computational systems have been demonstrated, including pressure-driven digital circuits $[15$, 16]. The Soft Matter Computer presents an attractive alternative computation system within the body of a soft robot and utilizes electro-fluidic coupling in an artificial vascular system [9]. These novel in-body control systems free future 
soft robots from reliance on conventional, rigid and nonbiodegradable silicon computing.

\subsection{Environmental compatibility}

As the number of robots in a population increase, and they are used in ever wider applications, the problem of resilience emerges. The goal of conventional robotics is to fabricate robots with as long a functional life as possible. This is in tension with the negative environmental impact of these robots when they reach the end of their productive lives. As the number of soft robots increase, resilience and post-life effects become increasingly important. To overcome this problem, biodegradability is important. Just as in nature, where all organic materials are recycled when an organism dies, biodegradable soft robots that degrade harmless to nothing are needed. To achieve this capability, biodegradable bodies must be developed, which encompass biodegradable actuators and sensors. Recently, soft actuators fabricated from biodegradable biopolymers have been demonstrated, including pneumatically [17] and electrically [18] driven structures. It is important to note that decomposition is not the same as safe degradation, as evidenced by the degradation of some plastics. In these cases, although the plastic has degraded, the constituent components still can have negative environmental impact. Environmental soft robotics, in contrast, must demonstrate a net neutral, or positive, environmental impact, considering whole-life-cycle and long-term environmental effects.

\subsection{Energy}

Conventional robotic systems have relied on stored electrical sources, batteries and $\mathrm{H}_{2} / \mathrm{O}_{2}$ fuel cells. These are normally charged at the beginning of a mission and can only be replenished when the robot returns to its base. Alternatively, energy can be harvested during operation through photovoltaic generation of electricity [4]. This is effective where light energy is plentiful but cannot be used in dark environments such as in deep oceans. Recently, alternative energy sources have been developed which gather energy from the environment. These include microbial fuel cells (MFCs), which metabolise organic matter and directly turn it into electrical energy [19]. The most striking use of MFCs in robots is shown in the EcoBot series of robots [20]. More recently Philamore et al. showed the RowBot, a soft materials-enhanced robot that can operate indefinitely in polluted environments and which can convert organic pollution into safe electrical energy [21]. There is also the potential to inoculate MFCs with microbial species which metabolise waste plastics, such as modified Pseudomonas strains [22], thereby reducing harmful environmental plastics. These show the potential of soft robots to operate autonomously and for long periods of time, delivering positive net environmental impact.

\subsection{Sustainability}

For robots to exist in potentially hazardous and delicate natural and artificial environments, they must be sustainable. This not only includes consideration of their environmental credentials (impact, cost, persistence, etc.), but also their ability to cope with damaging conditions. Rigid robots have limited ability to repair when damaged; it is challenging to repair a broken metal or rigid composite component. In contrast, soft robotics can be fabricated from materials which are robust to damage and which can self-heal. Self-healing is a property that is used by natural organisms to prolong their lives and to safely reach maturity. Examples include self-healing in Diels-Alder materials [23], and-more excitingly—self-healing in biodegradable polymers including konjac glucomannan gels [24]. While these employ molecular-level self-healing, a structural approach can also be taken, for example by employing capsule-based and vascular healing systems [25]. These more closely replicate the mechanisms found in nature, where processes including local clotting and fibroplasia are coordinated to close and repair tissue damage. Self-healing is a critical property for practical and long-living soft robots.

\subsection{Novel transduction}

Soft robotics has provided a fertile and inspirational ground for the development of unconventional actuation and sensing technologies. Traditional robotics has almost exclusively used electromagnetic motors and solenoids, which are dependent on complex electrical windings and, commonly, rare-earth magnetic materials. These are costly, constrain down-scaling of actuation and remain persistent in the natural environment long after they stop working. Nature, almost exclusively, does not exploit this electromagnetic-mechanical transduction principle, instead relying on chemo-mechanical energy conversion through muscles. Muscle systems extend from the nano-metre scale of the actin-myosin ratchet mechanism to the meter scale of skeletal muscle groups. The ability to scale actuation across over nine orders of magnitude is extremely attractive for future soft robotics. Artificial muscles, which aim to replicate, either implicitly or explicitly, natural muscular actuation, are a significant enabler of both physical and numerical scale-up of soft robotic systems [26]. One undesirable characteristic of soft transduction systems is their vulnerability to external disturbances. The challenge 
therefore is to maximise the accuracy of transduction (e.g. sensitivity and stationarity of soft sensors) while preserving their adaptability.

\section{Robots as organisms}

All soft robotics encompass one or more of the characteristics highlighted above. It is striking, however, to note that biological organisms exploit all of them. It is natural therefore to extend the concept of a soft robot to more closely resemble a biological organism. In this way, we not only focus on reproducing some of their effective capabilities and behaviours, but also overturn the conceptual restrictions inherent in the notion of a robot. A soft robotic organism includes the three high-level components of its biological counterpart, as shown in Fig. 2 in comparison with conventional rigid robots. There is a direct equivalence between the two, and recent soft robotic technologies enable the ready realisation of proto-robotic organisms:

Brain and control: The brain of an organism can be replicated by the all-soft control systems of the soft matter computer [9], combining centralised control with distributed computation akin to the peripheral nervous system. Additional computation and control can be devolved to the distal parts of the robot by adopting morphological computation techniques, which are particularly applicable to compliant bodies.

Soft body and artificial muscles: Soft artificial muscles and soft materials enable an all-soft body to be realised. In common with many biological organisms, an endoskeleton may be used to increase strength. Artificial muscles attach to passive tissue and the skeleton (if present) and extend throughout the body to provide locomotion and manipulation capabilities. They also contribute to internal homeostatic processes such as the throughput of organic material through the stomach. By fabricating the body and muscles from biodegradable materials, the whole robot can be made environmentally safe. Animals can even digest the robot with no ill effects. This is in stark contrast to conventional robots using persistent and toxic materials.

Artificial stomach and metabolism: The soft robotic organism can harvest organic materials (food and waste chemicals) from the environment, digesting them in an artificial stomach such as a microbial fuel cell [19]. This capability enables the robotic organism to survive indefinitely in environments where organic materials are plentiful, including in oceans and most terrestrial environments.

The three core features described above enable the reproduction of a wide range of essential high-level behaviours including graceful death, biodegradation, recycling and operation in very large numbers.

\subsection{Limitations and challenges}

The robotic organism is not a panacea and has a range of functional limitations and developmental challenges. The homeostatic processes that the robotic organism must maintain take energy and time. In some applications, this overhead is incompatible with a critical mission. For example, after an oil spill the immediate environmental risk may require the rapid deployment of conventional robots which can devote all their energy to the clean-up operation. In this case, the overhead of having to recover the non-biodegradable robots is balanced by the immediate environmental benefit.

Currently, several key capabilities in biological organisms cannot be replicated in robotic organisms. These include the ability to reproduce. All robots, including robotic organisms, must be manufactured using conventional methods. The ability for robotic organisms to reproduce will greatly enhance their scope of operations, but will require significant research. For example, to spawn a new robot organism,
Fig. 2 High-level equivalent components of biological organisms, conventional robots and soft robotic organisms

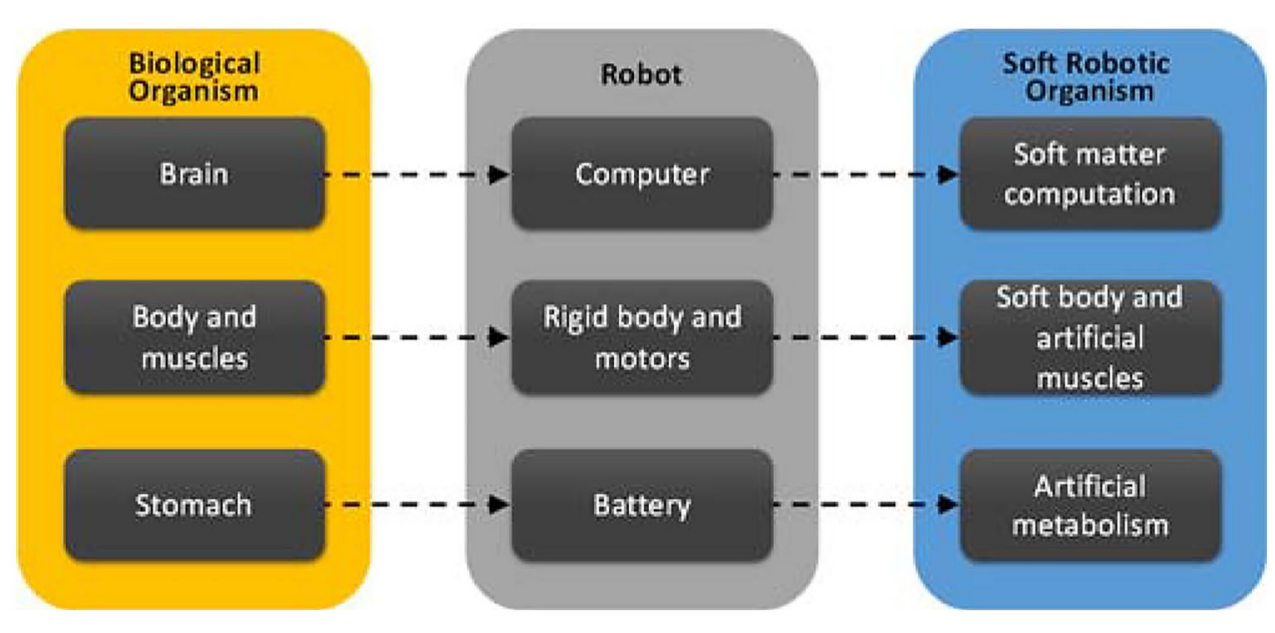


only materials that are available in the surrounding environment can be used. This is a significant and currently unsurmountable barrier to the autonomous reproduction of robotic organisms.

Soft robotic organisms still face major challenges. Although the constituent components are available (as described above) they have not yet been brought together to deliver a complete artificial organism. This integration, and parallel scale-up of capabilities, will be a major focus of research over the next decade.

\section{Conclusion}

Soft Robotic organisms represent a new paradigm in robotics. They can be fabricated and cooperate in populations greatly exceeding those of conventional robots, can degrade safely to nothing in vulnerable environments and can exist indefinitely by scavenging energy. The next challenge in this field is to combine the separate components and capabilities of soft robots to deliver a proto-organism. Following this, more sophisticated biological behaviours will be developed, including self-replication and reproduction. This will truly deliver a new world of 'living' soft robotic organisms.

Acknowledgements Rossiter is supported by EPSRC grants EP/ L015293/1, EP/M020460/1, EP/R02961X/1 and EP/S026096/1, and the Royal Academy of Engineering through the Chair in Emerging Technologies scheme.

Open Access This article is licensed under a Creative Commons Attribution 4.0 International License, which permits use, sharing, adaptation, distribution and reproduction in any medium or format, as long as you give appropriate credit to the original author(s) and the source, provide a link to the Creative Commons licence, and indicate if changes were made. The images or other third party material in this article are included in the article's Creative Commons licence, unless indicated otherwise in a credit line to the material. If material is not included in the article's Creative Commons licence and your intended use is not permitted by statutory regulation or exceeds the permitted use, you will need to obtain permission directly from the copyright holder. To view a copy of this licence, visit http://creativecommons.org/licenses/by/4.0/.

\section{References}

1. Nocks L (2006) The robot: the life story of a technology. Greenwood Publishing Group

2. Urhal P, Weightman A, Diver C, Bartolo P (2019) Robot assisted additive manufacturing: a review. Robot Comput Integr Manuf 59:335-345

3. Peters BS, Armijo PR, Krause C et al (2018) Review of emerging surgical robotic technology. Surg Endosc 32:1636-1655

4. Gao Y, Chien S (2017) Review on space robotics: toward top-level science through space exploration. Sci Robot 2(7):eaan5074

5. Asano Y, Okada J, Inaba M (2017) Design principles of a human mimetic humanoid: humanoid platform to study human intelligence and internal body system. Sci Robot 2(13):eaaq0899
6. Gravish N, Lauder GV (2018) Robotics-inspired biology. J Exp Biol 221:jeb138438

7. Katzschmann RK, DelPreto J, MacCurdy R, Rus D (2018) Exploration of underwater life with an acoustically controlled soft robotic fish. Sci Robot 3(16):eaar3449

8. Booth JW, Shah D, Case JC, White EL, Yuen MC, Cyr-Choiniere O, Kramer-Bottiglio R (2018) OmniSkins: robotic skins that turn inanimate objects into multifunctional robots. Sci Robot 3(22):eaat 1853

9. Garrad M, Soter G, Conn AT, Hauser H, Rossiter J (2019) A soft matter computer for soft robots. Sci Robot 4(33):eaaw6060

10. Philamore H, Ieropoulos I, Stinchcombe A, Rossiter J (2016) Toward energetically autonomous foraging soft robots. Soft Robot 3(4):186-197

11. Schuerle S, Soleimany AP, Yeh T, Anand GM, Häberli M, Fleming HE, Mirkhani N, Qiu F, Hauert S, Wang X, Nelson BJ, Bhatia SN (2019) Synthetic and living micropropellers for convectionenhanced nanoparticle transport. Sci Adv 5(4):eaav4803

12. Slavkov I, Carrillo-Zapata D, Carranza N, Diego X, Jansson F, Kaandorp JA, Hauert S, Sharpe J (2018) Morphogenesis in robot swarms. Sci Robot 3(25):eaau9178

13. Katzschmann RK, Thieffry M, Goury O, Kruszewski A, Guerra T-M, Duriez C, Rus D (2019) Dynamically closed-loop controlledsoft robotic arm using a reduced order finite element model with state observer. In: 2019 2nd IEEE international conference on soft robotics (RoboSoft), p 717-724

14. Na S, Qiu Y, Turgut AE, et al. (2020) Bio-inspired artificial pheromone system for swarm robotics applications. Adapt Behav. Accessed 3 June 2020

15. Rothemund P, Ainla A, Belding L, Preston DJ, Kurihara S, Suo Z, Whitesides GM (2018) A soft, bistable valve for autonomous control of soft actuators. Sci Robot 3(16):eaar7986

16. Mahon ST, Buchoux A, Sayed ME, Teng L, Stokes AA (2019) Soft robots for extreme environments: removing electronic control. In: 2019 2nd IEEE international conference on soft robotics (RoboSoft), p 782-787

17. Shintake J, Sonar H, Piskarev E, Paik J, Floreano D (2017) Soft pneumatic gelatin actuator for edible robotics. In: 2017 IEEE/RSJ international conference on intelligent robots and systems (IROS), p 6221-6226

18. Chambers L, Winfield J, Ieropoulos I, Rossiter JM (2014) Biodegradable and edible gelatine actuators for use as artificial muscles. SPIE: electroactive polymer actuators and devices (Vol. 9056). [90560B] Society of photo-optical instrumentation engineers (SPIE)

19. Winfield J, Chambers L, Stinchcombe A, Rossiter JM, Ieropoulos I (2014) The power of glove: soft microbial fuel cell for lowpower electronics. J Power Sources 249:327-332

20. Ieropoulos I, Greenman J, Melhuish C, Horsfield I (2010) EcoBot-III: A robot with guts. The 12th international conference on the synthesis and simulation of living systems, ALIFE 2010, p 733-740

21. Rossiter J, Philamore H, Stinchcombe A, Ieropoulos I (2015) Row-bot: an energetically autonomous artificial water boatman. In: 2015 IEEE/RSJ International Conference on Intelligent Robots and Systems (IROS), p 3888-3893

22. Espinosa MJC, Blanco AC, Schmidgall T, Atanasoff-Kardjalieff AK, Kappelmeyer U, Tischler D, Pieper DH, Heipieper HJ, Eberlein C (2020) Toward biorecycling: isolation of a soil bacterium that grows on a polyurethane oligomer and monomer. Front Microbiol 11:27

23. Terryn S, Brancart J, Lefeber D, Van Assche G, Vanderborght B (2017) Self-healing soft pneumatic robots. Sci Robot 2(9):eaan4268 
24. Song C, Lv Y, Qian K, Chen Y, Qian X (2019) Preparation of konjac glucomannan-borax hydrogels with good self-healing property and $\mathrm{pH}$-responsive behavior. J Polym Res 26:52

25. Willocq B, Odent J, Dubois P, Raquez J-M (2020) Advances in intrinsic self-healing polyurethanes and related composites. RSC Adv 10:13766-13782

26. Taghavi M, Helps T, Rossiter J (2018) Electro-ribbon actuators and electro-origami robots. Sci Robot 3(25):eaau9795
Publisher's Note Springer Nature remains neutral with regard to jurisdictional claims in published maps and institutional affiliations. 